\title{
An assessment of public transport features in urban and rural settlements in Malaysia
}

\begin{abstract}
This paper focuses on assessing the features of bus services offered in selected urban and rural settlements in Malaysia. Five definitions of public transport level of services (LOS) according to Transportation Research Board are adopted. Assessment of the LOS has been conducted using both quantitative and qualitative methods of primary and secondary data capture. With a sample of more than 1000 passengers surveyed, the demand side's perception and aspiration have been collected and analyzed based on the classification of LOS. The overall performance measured in urban and rural were LOS E, which is below the tolerable standard of LOS D. In analyzing the satisfaction levels among the urban and rural passengers, it was found that the majority of the respondents $(60.4 \%)$ were dissatisfied with the services in both urban and rural areas. Further recommendations are to bridge these gaps by increasing headways, locating designated bus stops or halts at strategic catchment points of alighting and dropping off as well as revised maintenance scheduling and consistent repair of the existing fleet. Finally, operators may also improve on-board comfort and convenient levels as aspired by the passengers. This can be commensurate by the willingness of passengers to pay higher fare rates for the increased quality of such services.
\end{abstract}

Keyword: Level of service (LOS); Passengers' satisfaction survey; Public bus service 